diverse towns in four Australian states (total population 72000 16-29-year-olds). To date, 27 towns have been randomised (13 intervention, 14 control). Intervention and control towns are similar: baseline chlamydia prevalence $(5.8 \%, 95 \%$ CI 4.4 to $7.5 \%$ vs $5 \%, 95 \%$ CI 3.9 to $6.3 \%$, individual response rate $63 \%$ ); past chlamydia testing rate in $16-29$-year-olds $(6.1 \%, 95 \%$ CI 5.8 to $6.4 \%$ vs $5.8 \%, 5.6$ to $6 \%$ ). After 3 months of the intervention in the first two towns the chlamydia testing rate was $10.9 \%$ (95\% CI $10.1 \%$ to $11.7 \%$; 691 people tested), a 160\% increase compared with the testing rate $(4.1 \%)$ in the year before the intervention.

Conclusions Our high recruitment rate shows that Australian GPs are willing to test for chlamydia. The baseline data show a high prevalence of chlamydia among young adults in the study towns. While testing rates are increasing, ongoing work is needed to get coverage up to levels that might reduce prevalence in intervention towns.

\section{9 IS HIV AND BLOOD BORNE VIRUS TESTING ACCEPTABLE AND INDICATED IN PSYCHIATRIC SETTINGS?}

doi:10.1136/sextrans-2012-050601a.9

${ }^{1} \mathrm{C}$ Sanger, ${ }^{*} 1 \mathrm{~J}$ Hayward, ${ }^{1} \mathrm{G}$ Patel, ${ }^{2} \mathrm{~A}$ Poots, ${ }^{2} \mathrm{C}$ Howe, ${ }^{1} \mathrm{~J}$ Green. ${ }^{1}$ Central North West London NHS FT, London, UK; ${ }^{2}$ NIHR CLAHRC for Northwest London, London, UK

Objective Studies in North America and Europe indicate that the prevalence of blood borne viruses (BBVs) are elevated in individuals with severe mental illness. No comparable data exists for the UK. We introduced the routine offer of testing for HIV, Hepatitis B and C into an inpatient psychiatric unit, where the local diagnosed HIV prevalence is 7.29/1000.

Method Psychiatric inpatients were approached at one central London hospital site, between April 2011 and February 2012 and offered routine BBV testing.

Results Of the first 100 patients offered a test $83 \%$ of patients approached were assessed to have mental capacity to provide informed consent for testing. $69 \%$ of patients offered BBV testing, accepted. The prevalence of HIV was found to be $3 \%$. One individual was newly diagnosed with HIV and transferred to specialist care. Overall, $18 \%$ of patients tested were found to have a newly diagnosed or previous infection with a BBV.

Conclusion It is acceptable to patients to be offered routine BBV screening in a psychiatric setting and the majority have capacity to consent; uptake rate is comparable to that seen in GUM clinics. HIV prevalence rate was found to be over four times higher than that of the local population. Given the elevated prevalence rates in psychiatric patients, there is a strong case for the wider introduction of routine testing in mental health settings. There is a need to systematically ascertain rates of infection in mental health patients across a range of geographical areas since the prevalence of BBVs appears to be higher than that in the local population.

\section{SHOULD SEXUAL HEALTH SERVICES PARTICIPATE IN THE HPV VACCINATION PROGRAMME? A NATIONAL SURVEY ASSESSING HPV VACCINATION UPTAKE IN YOUNG GUM ATTENDEES}

doi:10.1136/sextrans-2012-050601a.10

${ }^{1} \mathrm{R}$ Sacks, ${ }^{*}{ }^{2} \mathrm{~A}$ Robinson, ${ }^{1} \mathrm{D}$ Wilkinson. ${ }^{1}$ Imperial College Healthcare NHS Trust, London, UK; ${ }^{2}$ Central and North West London NHS Foundation Trust, London, UK, On behalf of the BASHH Adolescent Special Interest Group

Background In 2008, a schools-based HPV vaccination programme was introduced for girls aged 12-13, with an accelerated catch up programme for those aged 14-18. A significant impact on cervical cancer rates requires $80 \%$ uptake of three vaccinations, however in England the completion rate was 58\% in 2009/10.
Aim To compare HPV vaccination outcomes and prevalence of risk factors, associated with HPV acquisition and cervical cancer development, in young women attending GUM clinics with national data. Method An anonymous questionnaire was given to 13-19y/old women attending 19 participating GUM clinics from March to August 2011. Data were analysed using multivariate linear regression in SPSS Results 2247 questionnaires were completed (median respondent age 17). Compared to national data, respondents were more likely to be smokers ( $48 \%$ vs $12 \%$ of $15 \mathrm{y} /$ olds), have had coitarche aged $<16$ ( $52 \%$ vs $26 \%$ ), have had an STI previously $(29 \%$ vs $13 \%$ for $<16$ coitarche) or not be in education, employment or training (NEET) ( $8 \%$ vs $2 \%$ of $16 \mathrm{y} /$ olds). Of the $74 \%$ offered the vaccination, $81 \%$ accepted. Of those accepting the vaccination, $81 \%$ had all three injections. Of those who had $<3$ doses, $65 \%$ reported no active recall. Overall, $47 \%$ of all respondents had received all three vaccine doses. Completion rates were lower in London, non-white ethnicities, 17-19y/olds, NEETs, smokers and those with previous Chlamydia (all $\mathrm{p}<0.0001$ ).

Discussion The study population exhibited lower HPV vaccination completion rates than the national average, demonstrating that GUM attendees are a harder-to-reach group through current PCT delivery programmes. This population also exhibited higher rates of risk factors for HPV acquisition, highlighting GUM attendees as a priority target group for HPV vaccination. This data demonstrates the potential role of GUM clinics as supplementary HPV vaccination delivery sites, in targeting at-risk young women with low uptake of the HPV vaccination.

\section{HEALTH PROMOTIONAL MESSAGES IN SHORT MESSAGE SERVICE (SMS) FOLLOW-UP OF GU MEDICINE CLINIC DEFAULTERS; A TOOL TO IMPROVE SUBSEQUENT ATTENDANCE RATES?}

doi:10.1136/sextrans-2012-050601a.11

${ }^{1}$ E Rutland, ${ }^{*}{ }^{2} \mathrm{H}$ Roe, ${ }^{3} \mathrm{~A}$ Weaver. ${ }^{1}$ Western Sussex Hospitals NHS Trust, Western Sussex, UK; ${ }^{2}$ Southampton University Medical School, Southampton, UK; ${ }^{3}$ Solent Community NHS Trust

Background Patients who do not attend (DNA) booked GU medicine (GUM) clinics waste resources and may pose a public health risk through onward transmission of sexually transmitted infections. Short message services (SMS) appointment reminders improve attendance rates, however the impact of their use in patients who have already DNA'd is unknown. In addition, health promotional tools are frequently used to improve STI awareness however there is little evidence for their effect on GUM clinic attendance.

Aims To determine whether SMS follow-up of patients who DNA booked GUM appointments improves subsequent re-attendance rates and to assess the impact of inclusion of a health promotional message on re-attendance rates.

Methods This prospective randomised control study included all patients aged 16-30 yrs who DNA a booked GUM appointment during the 6 month study period. Subjects were randomised to: (1) no intervention; (2) SMS notification of the defaulted appointment and invitation to attend clinic; (3) as per arm 2 including a health promotional message about Chlamydia. All SMS were sent 1 week after the defaulted appointment. Patients re-booking or attending prior to this time were excluded. The proportion of patients who reattend within 4 weeks of the defaulted appointment were compared using the Fisher's Exact test.

Results 252 patients were included. $4.5 \%$ (4/88) in the control group re-attended clinic compared to $8.2 \%(7 / 85)$ receiving a text reminder $(p=0.36)$ and $15.2 \%(12 / 79)$ when a health promotional message $(p=0.032)$. None of those re attending in the control group were diagnosed with an STI compared to $29 \%$ in group 2 (Gonorrhoea and Chlamydia) and $25 \%$ in group 3 ( $2 \times$ Chlamydia and 1 Herpes) 